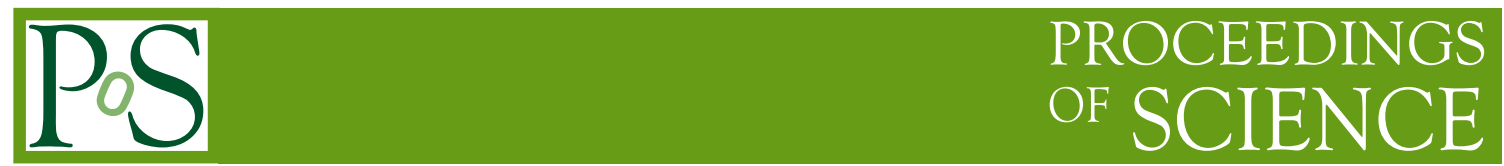

\title{
Micro Pattern Gaseous Detectors in High Energy Physics
}

\author{
Jochen Kaminski* \\ University of Bonn, Germany \\ E-mail: kaminski@physik.uni-bonn.de
}

An overview of new developments in Micro Pattern Gaseous Detectors (MPGDs) and their production techniques is given. Significant progress has been made in producing large area MPGDs with high quality. In particular, new types of Micromegas and Gas Electron Multipliers are discussed and some of their applications such as the time projection chamber for a linear collider are summarized. Also a new readout scheme based on highly pixelized CMOS-ASICs is presented.

35th International Conference of High Energy Physics - ICHEP2010,

July 22-28, 2010

Paris France

${ }^{*}$ Speaker. 


\section{Introduction}

Micro Pattern Gaseous Detectors (MPGDs) were first produced and described by A. Oed [1], who demonstrated functional Microstrip Gas Chambers (MSGCs) in 1988. A number of different detector types were tested in the following years, and two devices have proven to run reliably in a large variety of conditions: MICROMEsh GAseous Structures (Micromegas [2]) and Gas Electron Multipliers (GEMs [3]).

\section{Micromegas}

Micromegas detectors are two-stage parallel plate detectors where particles can ionize the gas in the large drift region (see figure 1a). Through the effect of the drift field (typically a few hundred $\mathrm{V} / \mathrm{cm}$ ), the charge then drifts towards the amplification gap through a grid. The strong electric field (typically some tens of $\mathrm{kV} / \mathrm{cm}$ ) in this $50-100 \mu \mathrm{m}$ thick region leads to an avalanche multiplication of the free charge. The signal is then collected by the anode readout structure that can be of various forms (strips, pads, pixels). A recent production technique is called bulk-Micromegas. A woven metallic grid is encapsulated between two layers of photoimageable film (see figure 1b). After the development of the film, the largest fraction of the insulator is washed out and only little pillars remain to keep the grid in place. A detailed study on optimization of these detectors with respect to the discharge probability has been conducted in the context of the COMPASS upgrade [4].

Microbulk-Micromegas are produced from thin kapton foils covered with copper on both sides (see figure 1c). The readout structure is patterned from one of the copper electrodes and can be made multi-layer by laminating additional layers of kapton and copper to this electrode. The mesh is patterned from the other electrode and the insulating pillars defining the gap between readout pads and the mesh are created by etching the original kapton layer from the mesh side. This procedure results in a very compact and easy to handle detector. In particular, the gap size determined by the thickness of the kapton can be well controlled and made very homogeneous. Therefore, the gas gain is very constant throughout the detector and the energy resolution is improved down to $11 \%$ FWHM for x-ray photons at $5.9 \mathrm{keV}$ [5].

Another interesting development is the use of highly pixelized CMOS-ASICs. With post processing methods similar to those of bulk-Micromegas the mesh is placed directly on top of the ASIC. An excellent alignment is possible and thus each readout pixel is placed directly below a hole in the mesh (see figure 2a). These devices are called InGrid and can detect individual primary electrons [6].
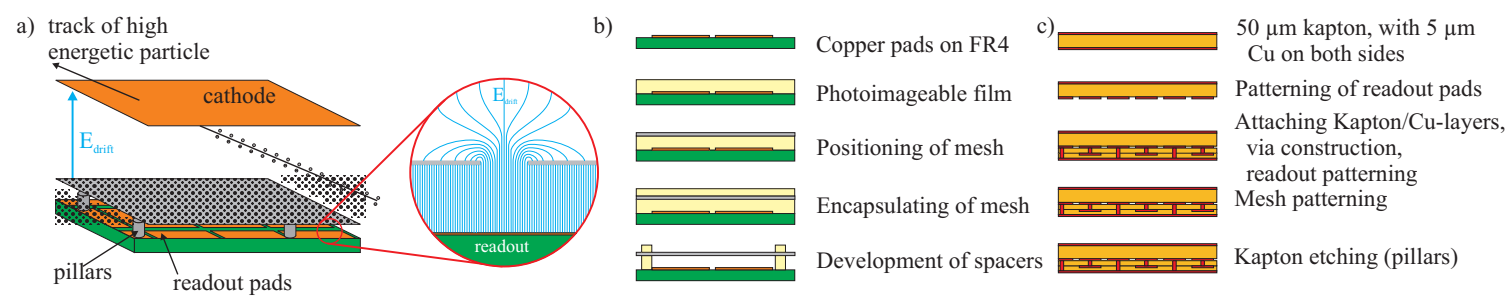

Figure 1: Micromegas: a) Schematic drawing of a Micromegas detector, b) production of bulk-Micromegas, c) production of microbulk-Micromegas. 

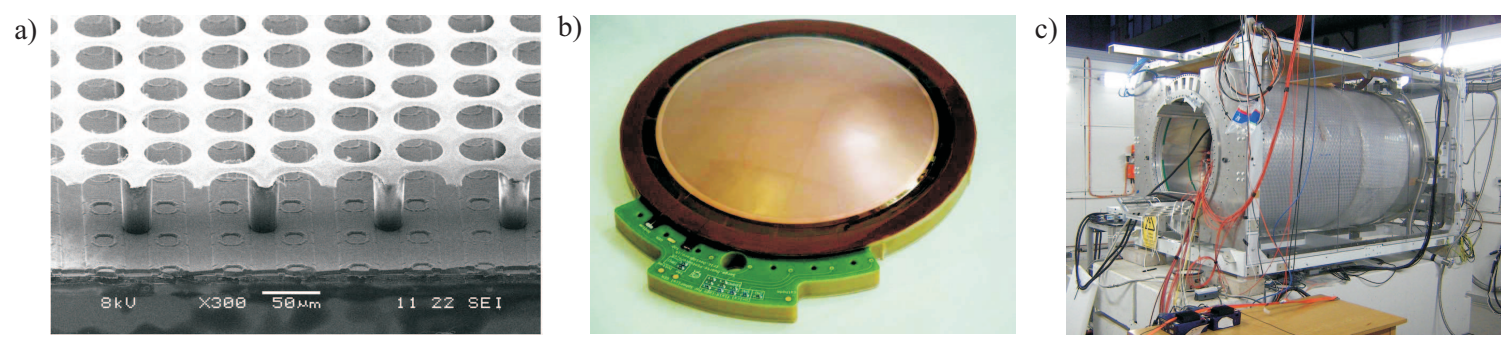

Figure 2: a) SEM picture of an InGrid [6], b) GEM with spherical shape [8], c) Large Prototype TPC of the EUDET collaboration inside the superconducting magnet on loan from KEK.

\section{GEM}

Gas Electron Multipliers consist of a thin insulating foil covered on both sides with an electrode. Holes of a typical size of 50-70 $\mu \mathrm{m}$ are etched with a pitch of typically $140 \mu \mathrm{m}$ in a hexagonal pattern through the complete sandwich structure. By applying a voltage difference to the two electrodes a strong electric field is generated in the holes and gas amplification takes place. During the production process two masks have to be aligned with a precision of $5 \mu \mathrm{m}$ or better. This requirement has limited the GEM area to about $40 \times 40 \mathrm{~cm}^{2}$. To overcome this restriction a new technique using only a single mask has been developed allowing the production of GEM areas up to $60 \times 200 \mathrm{~cm}^{2}$ [7].

Another novelty is the production of spherical GEMs with an opening angle of up to $106^{\circ}$ (see figure $2 b$ ). For this, standard GEM foils are placed in a vacuum and pressed at a temperature of more than $350^{\circ} \mathrm{C}$ for about $24 \mathrm{~h}$ over a mold [8]. These GEMs allow a parallax-free reconstruction of $\mathrm{x}$-ray images or high precision fixed target experiments.

Also, the readout of signals from a GEM stack with a highly pixelized CMOS-ASIC has been shown successfully [9]. Though signal areas are larger compared to InGrid detectors, the spatial resolution is comparable.

\section{Time Projection Chamber for a Linear Collider}

The Time Projection Chamber (TPC) for a Linear Collider has stringent requirements on its performance: The momentum resolution for example has to be better than $\delta\left(1 / p_{t}\right)=9 \cdot 10^{-5} c / \mathrm{GeV}$ resulting in a transverse spatial resolution of better than $100 \mu \mathrm{m}$ over the complete drift distance. This requirement can not be fulfilled by a standard wire readout of a classical TPC, but Micro Pattern Gas Amplification stages have to be used. The LCTPC collaboration is studying the various readout options. A large prototype with a maximum drift distance of $61 \mathrm{~cm}$ and an inner diameter of $72 \mathrm{~cm}$ has been set up at DESY, Hamburg [10]. The field cage is placed inside a superconducting solenoid, which can reach magnetic fields up to $B=1.25$ T. Particle tracks can be generated by the 1-6 GeV electron test beam of the DESY II accelerator. This detector serves to study various gas amplification stages, readout techniques and integration issues. Therefore, the endcap is designed to carry up to 7 interchangeable readout modules. Different groups have produced modules with Micromegas and pads, GEMs and pads, or GEMs with highly pixelized readout by CMOS chips. Despite the low magnetic field first measurements with the pad-based devices showed spatial res- 
olutions better than $140 \mu \mathrm{m}$. Extrapolating these results to high magnetic fields and longer drift distances proves that the requirements of a Linear Collider experiment can be fulfilled. Comparing the results of the pixel-based readout is more indirect, since the resolution is given for single electrons, whereas in pad-based devices the average of several tens of electrons is measured. However, first estimates indicate also good agreement with the pad-based results.

\section{Summary}

The development of Micro Pattern Gaseous detectors is a very active field. This is underlined by the formation of the CERN-based RD51 collaboration, which aims to facilitate the development of advanced gaseous detector technologies. Within 2 years of forming the collaboration already 73 institutes with more than 430 members have joined expressing the wide interest in the new technologies. A large part of the research is focused on improvements of Micromegas and GEM detectors. Only few of these developments could be introduced in this paper. Besides, one application example, a TPC for a Linear Collider, was given.

\section{Acknowledgment}

I would like to thank P. Colas, D. Neyret, E. Ferrer Ribas and J. Timmermans for providing me with material.

\section{References}

[1] A. Oed, Position sensitive detector with microstrip anode for electron multiplication with gases, Nucl. Instrum. Meth. A 263 (1988) 351.

[2] I. Giomataris, et al., MICROMEGAS: a high-granularity position-sensitive gaseous detector for high particle-flux environments, Nucl. Instrum. Meth. A 376 (1996) 29.

[3] F. Sauli, GEM: A new concept for gas amplification in gas detectors, Nucl. Instrum. Meth. A 386 (1997) 531.

[4] D. Neyret, et al., New pixelized Micromegas detector for the COMPASS experiment, JINST 4 (2009) P12004.

[5] J. Galan, et al., MICROMEGAS detectors in the CAST experiment, JINST 5 (2010) P01009.

[6] V. M. Blanco Carballo, et al. A Radiation Imaging Detector Made by Postprocessing a Standard CMOS Chip, IEEE Electron Device Letters 29 (2008) 585.

[7] S. Duarte Pinto, et al., Progress on large area GEMs, JINST 4 (2009) P12009.

[8] S. Pinto, et al. Spherical GEMs for parallax-free detectors, Nuclear Science Symposium Conference Record, 2009 IEEE.

[9] C. Brezina, et al., A Time Projection Chamber with triple GEM and pixel readout, JINST 4 (2009) P11015.

[10] T. Behnke, et al. A Lightweight Field Cage for a Large TPC Prototype for the ILC, arXiv:1006.3220v1, to be published in JINST. 